\title{
Pre-analytical quality control in hemostasis laboratories: visual evaluation of hemolysis index alone may cause unnecessary sample rejection
}

https://doi.org/10.1515/labmed-2018-0122

Received August 22, 2018; accepted February 6, 2019; previously published online March 22, 2019

\section{Abstract}

Background: Visual inspection is the most widespread method for evaluating sample hemolysis in hemostasis laboratories. The hemolysis index (HI) was determined visually (visual index, VI) and measured on an ACL TOP 750 (IL Werfen) system with a hemolysis-icterus-lipemia index (HIL) module. These values were compared with those measured on clinical chemistry systems Unicel DXC600 and AU680 and with quantitation of free-hemoglobin $(\mathrm{Hb})$ performed by a spectrophotometric measurement method (SMM).

Methods: The HI was measured in 356 sodium citrate plasma samples, 306 of which were visibly hemolyzed to varying degrees and 50 were not hemolyzed. The analytical performance of each method was evaluated.

Results: Linear regression analysis, calculated between SMM and the other systems in the study, returned coefficients of determination $\mathrm{r}^{2}=0.853$ (AU680), $\mathrm{r}^{2}=0.893$ (DXC600) and $\mathrm{r}^{2}=0.917$ (ACL TOP 750). An $\mathrm{r}^{2}=0.648$ was obtained for linear regression analysis between VI and ACL TOP 750. In addition, ACL TOP 750 showed an excellent correlation in multivariate analysis $\left(r^{2}=0.958\right)$, showing good sensitivity (0.939) and specificity (0.934) and a diagnostic accuracy of $94 \%$. By comparison,

\footnotetext{
*Correspondence: Dr. Simona Storti, U.O. Medicina di Laboratorio, Ospedale del Cuore G. Pasquinucci, Fondazione Toscana Gabriele Monasterio, Via Aurelia Sud, 54100 Massa, Italy, Tel.: +360585 483652, E-Mail: storti@ftgm.it Elena Battipaglia and Maria Serena Parri: U.O. Medicina di Laboratorio, Ospedale del Cuore G. Pasquinucci, Fondazione Toscana Gabriele Monasterio, Massa, Italy

Andrea Ripoli: U.O. Bioingegneria, Fondazione Toscana Gabriele Monasterio, Pisa, Italy

Stefania Lombardi and Giovanna Andreani: S.S.D Laboratorio Analisi Chimico-Cliniche e Microbiologia Molecolare, Nuovo Ospedale delle Apuane, Massa, Italy
}

DXC600 and AU680 showed coefficients of determination of 0.945 and 0.923 , respectively. A cut-off was set at 0.15 $\mathrm{g} / \mathrm{L}$ free- $\mathrm{Hb}$, as determined by the automated method, such that any hemostasis samples measuring above this threshold should not be analyzed. Based on this criterion, samples were classified as accepted or rejected, and the number of samples discarded during VI or ACL TOP 750 measurements was compared.

Conclusions: This study confirmed that hemostasis laboratories should consider introducing an objective, automated and standardized method to check samples for hemolysis. By relying solely on visual inspection, up to $50 \%$ of samples could be unnecessarily rejected. The ACL TOP 750 system demonstrated a satisfactory analytical performance, giving results comparable to those of the reference method.

Keywords: hemolysis index; hemostasis laboratory; preanalytical quality control.

Brief summary: In the hemostasis laboratory, it is necessary to use objective and standardized systems to assess sample hemolysis. We found that visual inspection alone might cause about $50 \%$ of samples to be discarded, $35 \%$ of which could have been processed if an automatic method had been used. The automatic hemolysis-icteruslipemia index (HIL) check performed on an ACL TOP 750 system demonstrated a satisfactory analytical performance and a good correlation with the reference method, the spectrophotometric measurement of free-hemoglobin $(\mathrm{Hb})$.

\section{Introduction}

A large amount of information is generated daily in clinical laboratories and this can potentially influence clinical decisions. To assure the validity of such information, quality control is essential at all stages of the analytical process, including the pre-analytical phase [1]. 
Pre-analytical activities, especially those directly connected with blood sample collection, handling and transportation, are the most vulnerable steps throughout the testing process [2]. Several guidelines and recommendations have been published for minimizing the impact of pre-analytical variables on the quality of test results [1-4]. In vitro hemolysis reflects a generalized process of cell damage that occurs during phlebotomy, being more frequent in patients with fragile veins, and also resulting from excessive vacuum aspiration, prolonged venous stasis or the use of excessively narrow needles [3]. When red blood cells break, hemoglobin $(\mathrm{Hb})$ and other intracellular components are released into the serum or plasma causing biological, chemical and spectrophotometric interference and sometimes invalidating the test results [2]. Most current testing guidelines for plasma-based coagulation assays recommend samples to be rejected when hemolysis is clearly visible [5]. Moreover, several scientific studies suggest that the influence of hemolysis on test results can vary widely. Depending on the type of analytical method (i.e. photo-optical or mechanical method), certain tests can be more affected than others by interference from $\mathrm{Hb}$, while some assays remain unaffected [6-8]. Analysis of unsuitable specimens can lead to unreliable results, which ultimately can affect patient safety [2]. Therefore, every laboratory should focus on continuously monitoring the quality of samples $[9,10]$.

Several non-automated spectrophotometric assays for free-Hb have been described. The method described by Fairbanks et al. [11] is based on an algorithm using absorbances measured at three wavelengths.

Usually in clinical chemistry laboratories, automated systems enable an assessment of levels of hemolysis by measuring absorbance or reflectance at $\geq 2$ different wavelengths, preferably at absorbance peaks of the specific substance. $\mathrm{Hb}$ absorbs light at wavelengths between 340 and $440 \mathrm{~nm}$ and between 540 and $580 \mathrm{~nm}$ [10]. Starting with the absorbances, these automated systems apply proprietary and undisclosed algorithms to quantify free- $\mathrm{Hb}$ in $\mathrm{g} / \mathrm{L}$; the systems then group the results into classes (from 1 to 10 ) in a semi-quantitative way. Unfortunately, individual systems use different classifications of the degree of hemolysis, making the comparison of results difficult [12].

Unlike clinical chemistry laboratories, the use of automated systems in hemostasis laboratories to measure hemolysis is not so widespread. Instead, most use visual inspection of individual specimens by laboratory personnel [10, 13]. Luksic et al. [14] described a method of visual assessment of hemolysis for plasma or serum samples after centrifugation in a clinical chemistry laboratory.
According to this procedure, technicians compare samples with a visual scale, using eight reference tubes containing different known concentrations of free-Hb. This method is easy, inexpensive and quick; however, the results are subject to operator variation $[9,14]$.

In hemostasis laboratories, the ACL TOP 750 system has been equipped with a pre-analytical module for plasma indices, allowing an assessment of the level of hemolysis, icterus and lipemia (HIL) in plasma samples $[13,15,16]$.

Only a few reports have thoroughly evaluated the analytical performance of automated coagulation tests and the consequences of using sample visual inspection alone in the pre-analytical process [9, 13-16].

The aim of the present study was to evaluate the technical performance of the pre-analytical HIL check module on ACL TOP 750, as compared to the hemolysis indices (HIs) measured by the automated chemistry systems Unicel DXC600 and AU680 and by the spectrophotometric method for quantitative assessment of free- $\mathrm{Hb}$, as described by Fairbanks et al. [11], and chosen as the reference method. The difference between the number of hemolyzed samples identified by visual inspection, automated methods and standardized procedures was also calculated.

\section{Materials and methods}

\section{Study design}

To study the analytical ability of different systems to discriminate and classify different levels of hemolysis, we initially used samples known to be hemolyzed. For this purpose, we selected 306 samples that were visibly hemolyzed to different degrees. HI was also measured in 50 samples that did not show visible hemolysis, representing a negative control group.

Blood was collected in $3.5 \mathrm{~mL}$ sodium citrate Vacutainer tubes (9NC buffered citrate 3.2\%, $0.109 \mathrm{M}$; Vacutest Kima s.r.l., Arzegrande, Italy). Whole blood samples were centrifuged at $1500 \mathrm{~g}$ for $15 \mathrm{~min}$ at room temperature, according to the sample preparation protocol for coagulation analysis [17]. Free-Hb was measured in plasma using ACL TOP 750 (Instrumentation Laboratory, Werfen, Bedford, MA, USA), spectrophotometric measurement method (SMM), Unicel DXC600 and AU680 (Beckman Coulter Inc., Fullerton, CA, USA). HI was also determined visually according to the method described by Luksic et al. [14], comparing each sample with a visual scale [14]. Visual scoring was performed by only one technician to 
reduce operator variability [9]. The visual chart by Luksic et al. included eight tubes with increasing concentrations of free- $\mathrm{Hb}$, labeled $1-8$, with samples $2-8$ being classified as hemolyzed [14]. For our work, we only used the first four references: tube 1 , free- $\mathrm{Hb}<0.1 \mathrm{~g} / \mathrm{L}$; tube $2,0.1<$ free$\mathrm{Hb}<0.5 \mathrm{~g} / \mathrm{L}$; tube 3, $0.5<$ free- $\mathrm{Hb}<1 \mathrm{~g} / \mathrm{L}$; and tube 4 , $1<$ free- $\mathrm{Hb}<2 \mathrm{~g} / \mathrm{L}$.

The analytical performance of the different assay methods was evaluated. Assay reproducibility was studied in accordance with the Clinical and Laboratory Standards Institute (CLSI) EP5-A2 protocol [18]. The limit of blank (LoB), limit of detection (LoD) and limit of quantification (LoQ) were calculated using the CLSI EP17-A protocol [19].

Ethical approval was obtained from the Internal Committee, located in Heart Hospital, Massa, Italy. Anonymous plasma from healthy donors and patients, leftover after routine analysis was used in the study. Informed consent was obtained for this analysis of donated residual biological material.

\section{Analyzers}

\section{Determination of hemolysis index on the DXC600 system}

The Unicel DXC600 system (Beckman Coulter Inc., Fullerton, CA, USA) was a clinical chemistry analyzer designed for labs with low work volumes (approximately 100-500 chemistry samples per day). The serum index function, in conjunction with a specific reagent (Synchron Systems DIL1 cartridge ref. 467826; Beckman Coulter Inc., Fullerton, CA, USA), was intended for the semi-quantitative determination of icterus (bilirubin), hemolysis ( $\mathrm{Hb})$ and lipemia in serum or plasma. A precise volume of sample $(14 \mu \mathrm{L})$ was injected in a cuvette containing $200 \mu \mathrm{L}$ of buffer. The ratio used was one part sample to 14.3 parts reagent. The system measured absorbance at 340, 410, 470, 600 and $670 \mathrm{~nm}$ and solved a set of equations to determine the response for each index. HI was expressed in 11 categories (from 0 to 10 ).

\section{Determination of hemolysis index on the AU680 system}

The AU680 system (Beckman Coulter Inc., Fullerton, CA, USA) was designed for middle to large-sized laboratories and hospitals. The analyzer could detect potential interfering substances by means of a semi-quantitative method. Patient samples $(1.6 \mu \mathrm{L})$ were diluted with $20 \mu \mathrm{L}$ of lipemia/ turbidity, icterus and hemolysis (LIH) reagent (System Reagent OSR62166; Beckman Coulter Inc., Fullerton, CA, USA) and absorbance was measured at six wavelengths
(410, 480, 570, 600, 660, $800 \mathrm{~nm})$. If one or more chromogens were present in a sample at a potentially interfering concentration, flags were generated that characterized its type (lipemia/turbidity, LIP; bilirubin, TIC; and Hb, HEM) and approximate concentration $(+,++,++++)$.

\section{Determination of hemolysis index on the ACL TOP 750 system}

The ACL TOP family was a complete line of fully automated hemostasis testing systems equipped with a photooptical clot-detection unit. They could determine HIL by assessing the levels of $\mathrm{Hb}$, total bilirubin and lipemia in a sample. HIL was determined by optical absorbance measurements at three different wavelengths of the diluted sample, $405 \mathrm{~nm}$ for free-Hb and total bilirubin, $535 \mathrm{~nm}$ for turbidity and free- $\mathrm{Hb}$ and $671 \mathrm{~nm}$ for turbidity. Measurement of the absorbances created three equations, which could then be solved mathematically to determine the levels of the three different interfering substances. The plasma indices were generated in $\mathrm{g} / \mathrm{L}$ for $\mathrm{Hb}, \mathrm{mg} / \mathrm{dL}$ for bilirubin and milli-absorbances (mAbs) for turbidity [13].

\section{Manual spectrophotometric measurement of free- $\mathrm{Hb}$}

The method used to quantify free-Hb by spectrophotometry was derived from the one described by Fairbanks et al. [11], with a modification of the sample matrix to sodium citrate plasma instead of ethylenediaminetetraacetic acid (EDTA) plasma. Plasma citrate samples, used for coagulation tests, were centrifuged at $2000 \mathrm{~g} \times 10 \mathrm{~min}$ at room temperature, to remove any residual red blood cells. All specimens were diluted 11-fold in $0.942 \mathrm{~mol} / \mathrm{L} \mathrm{Na}_{2} \mathrm{CO}_{3}$ before absorbance measurements. Absorbances were measured at 415, 450 and $700 \mathrm{~nm}$. All spectrophotometric measurements were performed using an Ultrospec 2000 spectrophotometer (Pharmacia Biotech Inc., Piscataway, NJ, USA). Total free-Hb was calculated according to the equation [11]:

$$
\mathrm{Hb}(\mathrm{g} / \mathrm{L})=\left(154.7 * \mathrm{~A}_{415}-130.7 * \mathrm{~A}_{450}-123.9 * \mathrm{~A}_{700}\right) / 100
$$

where $A_{415}, A_{450}$ and $A_{700}$ were the absorbance values measured at 415, 450 and $700 \mathrm{~nm}$, respectively.

\section{Statistical analysis}

Data were analyzed using Statistical Discovery Software JMP, version 5.0 (SAS Institute Inc., Cary, NC, USA). Linear regressions, Bland-Altman plots, reproducibility, LoD, LoB, LoQ, sensitivity and specificity were calculated. 


\section{Results}

\section{Samples}

The samples were collected over a period of 6 months. The 306 visibly hemolyzed samples were from 116 males (38\%) and 190 females (62\%), mean age $74 \pm 18$ years (mean \pm standard deviation [SD]; range 5-101 years). Thirty-one percent of the samples came from emergency units, $13 \%$ from hospital departments, $26 \%$ from local blood collection centers and 30\% from home visits. Free-Hb ranged from 0.24 to $4.05 \mathrm{~g} / \mathrm{L}$ (mean $0.86 \pm 0.58$ ). The 50 samples of the control group from healthy blood donors had a mean free-Hb of $0.031 \mathrm{~g} / \mathrm{L} \pm 0.025$. No overlap between measurements from hemolyzed samples and those of the control group (non-hemolyzed) was observed for any method (Figure 1). All data were normally distributed.

\section{Evaluation of analytical methods}

\section{Spectrophotometric measurement method (SMM)}

Assay reproducibility was evaluated by repeatedly measuring two pools of plasma citrate samples at two different hemolysis levels, containing a mean $\mathrm{Hb}$ of $0.477 \pm 0.0064 \mathrm{~g} / \mathrm{L}$ (sample A) and $1.363 \pm 0.0123 \mathrm{~g} / \mathrm{L}$ (sample B), as measured by spectrophotometer. Withinrun and total coefficients of variation (CVs\%) were $1.28 \%$ and $2.10 \%$ for the lower concentration sample $\mathrm{A}$, and $0.79 \%$ and $1.91 \%$ for the higher concentration sample B, respectively (Table 1 ).

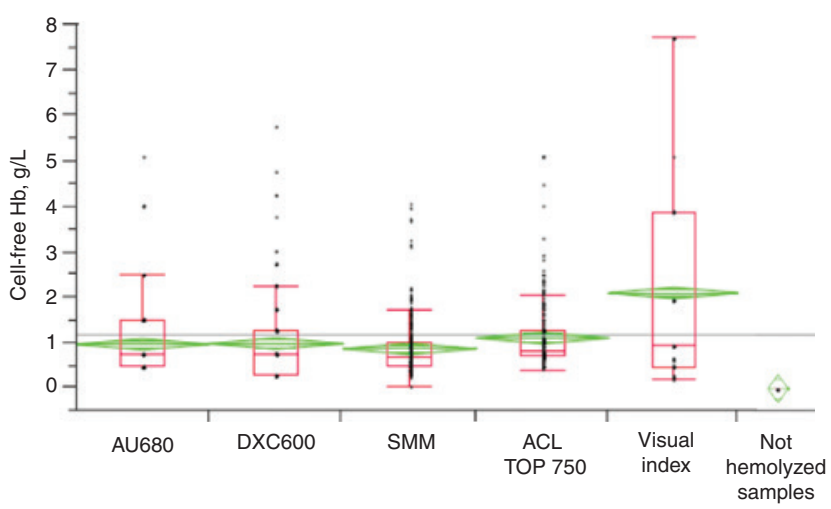

Figure 1: Free-Hb in 306 visibly hemolyzed samples, measured by SMM, ranging from 0.24 to $4.05 \mathrm{~g} / \mathrm{L}$ (mean $0.86 \pm 0.58$ ). Fifty not visibly hemolyzed samples were analyzed (mean free- $\mathrm{Hb}$ $0.031 \mathrm{~g} / \mathrm{L} \pm 0.025$ ). No overlap between hemolyzed and nonhemolyzed samples was observed for any method.
Table 1: Spectrophotometric method for free- $\mathrm{Hb}$ measurement.

\begin{tabular}{lrrrrr}
\hline Sample & $\mathbf{n}$ & $\begin{array}{r}\text { Free-Hb } \\
\text { mean, g/L }\end{array}$ & $\begin{array}{r}\text { Free-Hb standard } \\
\text { deviation, g/L }\end{array}$ & $\begin{array}{r}\text { CV\% } \\
\text { within run }\end{array}$ & $\begin{array}{r}\text { CV\% } \\
\text { tot }\end{array}$ \\
\hline A & 20 & 0.477 & 0.0064 & 1.28 & 2.10 \\
B & 20 & 1.363 & 0.0123 & 0.79 & 1.91 \\
\hline
\end{tabular}

Within-run and total CVs\% were evaluated according to the CLSI EP5-A2 protocol by repeatedly measuring two pools of plasma citrate samples with two different degrees of hemolysis.

LoB and LoQ were calculated by measuring a solution containing no free- $\mathrm{Hb}$ (saline solution), 60 times in three different runs, obtaining mean absorbance units of $0.002 \pm 0.002$. A curve was plotted, taking into account 306 free- $\mathrm{Hb}$ values in the concentration range of $0-2 \mathrm{~g} / \mathrm{L}$ $\left(\mathrm{A}_{415}=0.101+0.745 \mathrm{Hb}_{(\mathrm{g} / \mathrm{L})}\right)$. From this curve, the absorbance of a solution containing no free-Hb was extrapolated and a LoB value of $0.0044 \mathrm{~g} / \mathrm{L}$ was then calculated.

The LoD value $(0.0212 \mathrm{~g} / \mathrm{L})$ was calculated using the formula:

$$
\mathrm{LoD}=\mathrm{LoB}+1.645 \mathrm{SD}
$$

where SD $(0.01025 \mathrm{~g} / \mathrm{L})$ was estimated from the distribution of free-Hb values measured in a plasma sample of low free- $\mathrm{Hb}$ concentration $(\mathrm{Hb}=0.05 \mathrm{~g} / \mathrm{L})$ (Figure 2 ).

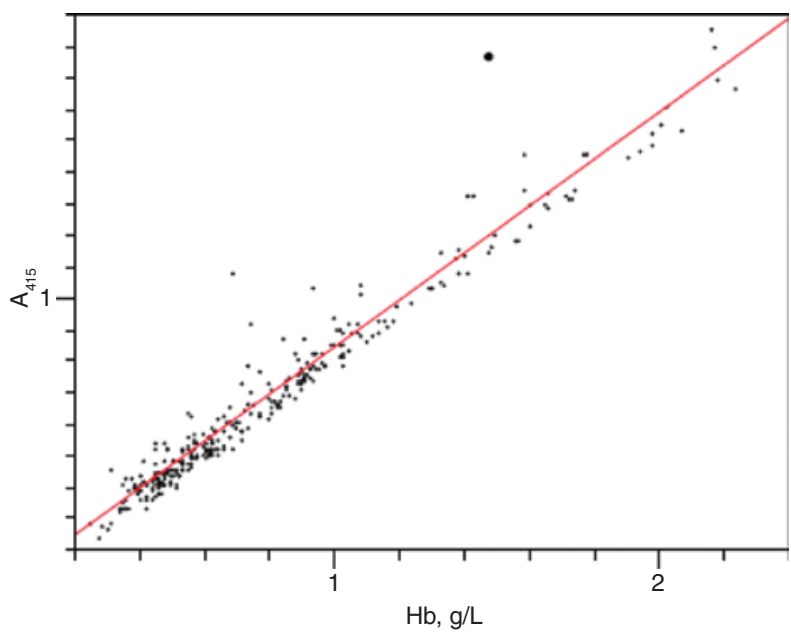

Figure 2: Limits of blank ( $L O B)$ and detection (LoD) were calculated according to the CLSI EP17-A protocol.

A solution containing no free- $\mathrm{Hb}$ (saline solution) was measured 60 times in three different runs. A mean absorbance value of $0.002 \pm 0.002$ was obtained. A curve of absorbance at $415 \mathrm{~nm}$ against free- $\mathrm{Hb}_{(\mathrm{g} / \mathrm{L})}$ was constructed, taking into account 306 free- $\mathrm{Hb}$ values in the range of concentration between 0 and $2 \mathrm{~g} / \mathrm{L}$ $\left(\mathrm{A}_{415}=0.101+0.745 \mathrm{Hb}_{(\mathrm{g} / \mathrm{L})}\right)$. From this curve, the absorbance of a solution containing no free- $\mathrm{Hb}$ was extrapolated and a LoB value of $0.0044 \mathrm{~g} / \mathrm{L}$ was then calculated. 


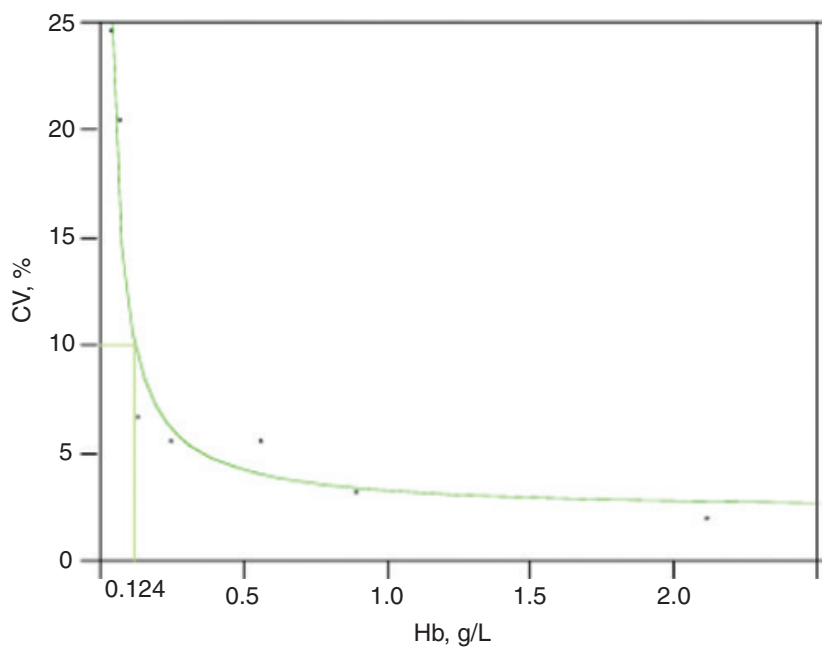

Figure 3: Limit of quantification ( $L O Q$ ) for free- $\mathrm{Hb}$, measured by the spectrophotometric method, was estimated according to the CLSI EP17-A protocol.

Six pools from sodium citrate plasma samples of low free- $\mathrm{Hb}$ concentration were prepared (from three different patients) and analyzed 40 times in five different runs, to estimate the total error (CV\%). The imprecision profile was then drawn up, plotting the mean concentration for each pool on the $\mathrm{x}$-axis and the corresponding CV\% on the $y$-axis. A LoQ $=0.124 \mathrm{~g} / \mathrm{L}$ at $10 \% \mathrm{CV}$ was obtained.
No analyzed plasma samples showed free- $\mathrm{Hb}$ values under the LoB.

LoQ was calculated by measuring six sodium citrate plasma pools (from three different patients), containing low concentrations of free- $\mathrm{Hb}$, analyzed 40 times in five different runs, to estimate the total $\mathrm{CV} \%$ error. An imprecision profile was then drawn up, plotting the mean concentration for each pool on the $\mathrm{x}$-axis and the corresponding CV\% on the y-axis. A $\mathrm{LoQ}=0.124 \mathrm{~g} / \mathrm{L}$ at $10 \% \mathrm{CV}$ was obtained (Figure 3).

The linearity of the method was evaluated by assaying serial dilutions of a sodium citrate plasma sample containing an original theoretical value of about $10 \mathrm{~g} / \mathrm{L}$ free- $\mathrm{Hb}$, down to a value close to the LoQ $(0.124 \mathrm{~g} / \mathrm{L})$. This created a linear regression between absorbance at $415 \mathrm{~nm}$ and the theoretical value of free- $\mathrm{Hb}\left(\mathrm{A}_{415}=0.023+0.656 \mathrm{Hb}_{(\mathrm{g} / \mathrm{L})}\right.$, coefficient of determination, $\left.r^{2}=0.999\right)$ and between the measured value of free- $\mathrm{Hb}$ and the theoretical $\mathrm{Hb}$ value (measured $\mathrm{Hb}_{(\mathrm{g} / \mathrm{L})}=-0.012+0.888 \mathrm{Hb}_{(\mathrm{g} / \mathrm{L})}, \mathrm{r}^{2}=0.998$; Figure 4). This method had originally been described on EDTA plasma samples [11]. When the linearity obtained using sodium citrate plasma samples was compared with one verified

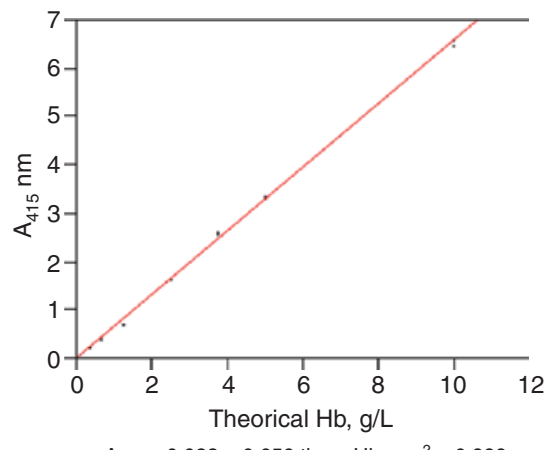

$A_{415}=0.023+0.656$ theor $\mathrm{Hb}_{(g / L)} r^{2}=0.999$

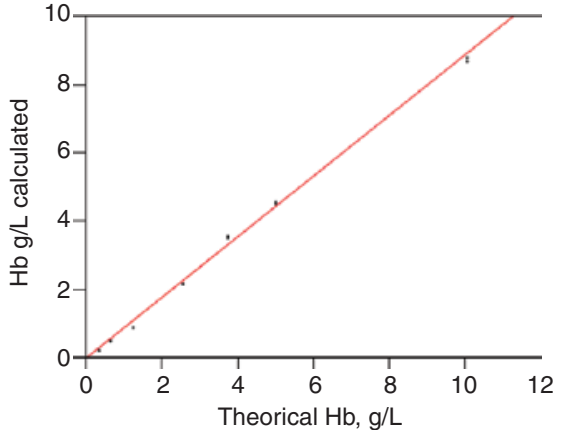

Calc $\mathrm{Hb}_{(\mathrm{g} / \mathrm{L})}=-0.012+0.888$ theor $\mathrm{Hb}_{(\mathrm{g} / \mathrm{L})} \mathrm{r}^{2}=0.998$

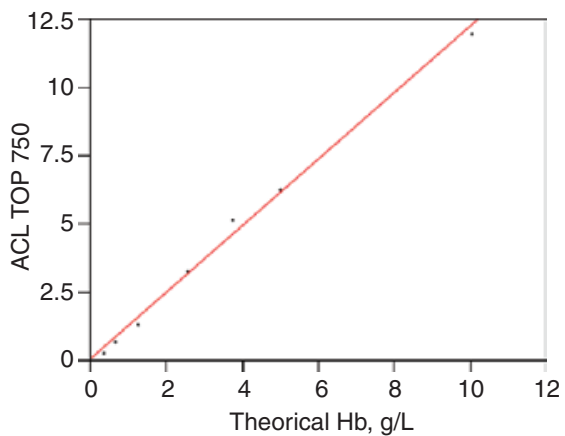

ACL TOP $750 \mathrm{HI}=0.0861 .22$ theor $\mathrm{Hb}_{(\mathrm{g} / \mathrm{L})} \mathrm{r}^{2}=0.995$
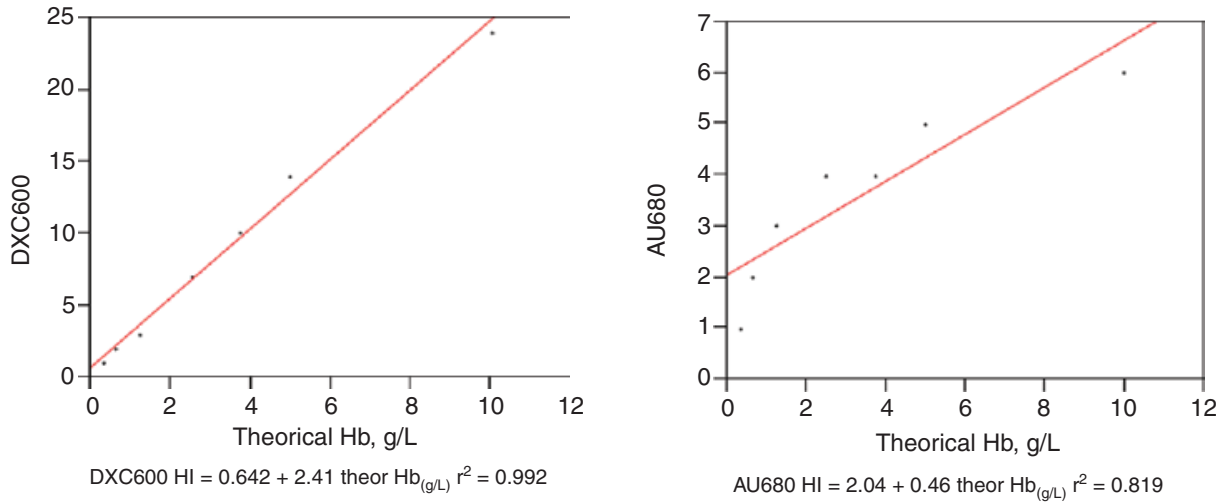

Figure 4: The linearity of all methods was evaluated by serial dilutions of a plasma sodium citrate sample containing an original theoretical value of about $10 \mathrm{~g} / \mathrm{L}$ free- $\mathrm{Hb}$, down to a value close to the LoQ, as calculated by the spectrophotometric method. 
for EDTA plasma samples, very similar curves for the two different kinds of plasma matrixes were obtained (data not shown).

\section{DXC600 and AU680 Beckman HI assay}

As the HI obtained from DXC600 and AU680 was automatically expressed as a score and was not a continuous value, LoB, LoD and LoQ for these methods could not be evaluated.

The linearity of HI measured on the DXC600 system was evaluated by serial dilutions of a sample originally containing about $10 \mathrm{~g} / \mathrm{L}$ free- $\mathrm{Hb}$, down to a value near the spectrophotometric LoQ $(0.124 \mathrm{~g} / \mathrm{L}$; DXC600 $\mathrm{HI}=0.642+2.41 \mathrm{Hb}_{(\mathrm{g} / \mathrm{L})}, \mathrm{r}^{2}=0.992$; Figure 4).

The linearity of HI scores obtained on the AU680 system was evaluated by serial dilutions of a sodium citrate plasma sample originally containing about $10 \mathrm{~g} / \mathrm{L}$ free- $\mathrm{Hb}$, down to a value near the spectrophotometric LoQ (AU680 HI=2.04 + $0.46 \mathrm{Hb}_{(\mathrm{g} / \mathrm{L})}, \mathrm{r}^{2}=0.819$; Figure 4).

Table 2: ACL TOP 750 HIL check.

\begin{tabular}{lrrrrr}
\hline Sample & $\mathbf{n}$ & $\begin{array}{r}\text { Free-Hb } \\
\text { mean, g/L }\end{array}$ & $\begin{array}{r}\text { Free-Hb standard } \\
\text { deviation, g/L }\end{array}$ & $\begin{array}{r}\text { CV\% } \\
\text { within run }\end{array}$ & $\begin{array}{r}\text { CV\% } \\
\text { tot }\end{array}$ \\
\hline A & 20 & 0.72 & 0.01 & 2.05 & 2.91 \\
B & 20 & 1.79 & 0.03 & 3.23 & 3.41 \\
\hline
\end{tabular}

Within-run and total CVs (\%) evaluated according to the CLSI EP5-A2 protocol by repeatedly measuring two pools of plasma citrate samples at two different degrees of hemolysis.

\section{ACL TOP 750 HIL check}

The reproducibility of the $\mathrm{HI}$ results obtained on the ACL TOP 750 system was evaluated in accordance with the CLSI protocol EP5-A2, repeatedly measuring two pools, A and B, of plasma citrate samples at two different degrees of hemolysis. HIL check on ACL TOP 750 showed that A and B contained a mean free-Hb of $0.72 \pm 0.01 \mathrm{~g} / \mathrm{L}$ and $1.79 \pm 0.03 \mathrm{~g} / \mathrm{L}$, respectively. Within-run and total CVs\% were $2.05 \%$ and $2.91 \%$ for low free-Hb concentration sample A, and 3.23\% and 3.49\% for high free-Hb concentration sample $\mathrm{B}$, respectively (Table 2).

It was not possible to calculate the LoQ using the CLSI EP17-A protocol, because the system always provided the same results (i.e. 0) if free- $\mathrm{Hb}$ in the sample was at low concentration, thus giving a $\mathrm{CV} \%$ for low concentrations of 0 . For the same reason, it was not possible to measure LoB.

The linearity of the HIL check was evaluated by serial dilutions of a sample originally containing about $10 \mathrm{~g} / \mathrm{L}$ free- $\mathrm{Hb}$, down to a value near the spectrophotometric LoQ $\left(0.124 \mathrm{~g} / \mathrm{L}\right.$; ACL TOP $750 \mathrm{HI}=0.086+1.222 \mathrm{Hb}_{(\mathrm{g} / \mathrm{L})}, \mathrm{r}^{2}=0.995$; Figure 4).

A good correspondence was observed between the free- $\mathrm{Hb}_{(\mathrm{g} / \mathrm{L})}$ of the serial dilutions measured on the ACL TOP 750 system and values of free- $\mathrm{Hb}_{(\mathrm{g} / \mathrm{L})}$ obtained from the DXC600 system $\left(\mathrm{Hb}_{(\mathrm{g} / \mathrm{L})}\right.$ ACL TOP $750=-0.04+1.01$ $\mathrm{Hb}_{(\mathrm{g} / \mathrm{L})}$ DXC600, $\left.\mathrm{r}^{2}=0.999\right)$. However, an unsatisfactory correspondence was found between the values of $\mathrm{Hb}_{(\mathrm{g} / \mathrm{L})}$ from the ACL TOP 750 system and those derived from scores on the AU680 system $\left(\mathrm{Hb}_{(\mathrm{g} / \mathrm{L})}\right.$ ACL TOP $750=-0.49+1.70 \mathrm{Hb}_{(\mathrm{g} / \mathrm{L})}$ AU680, $r^{2}=0.866$; Figure 5).
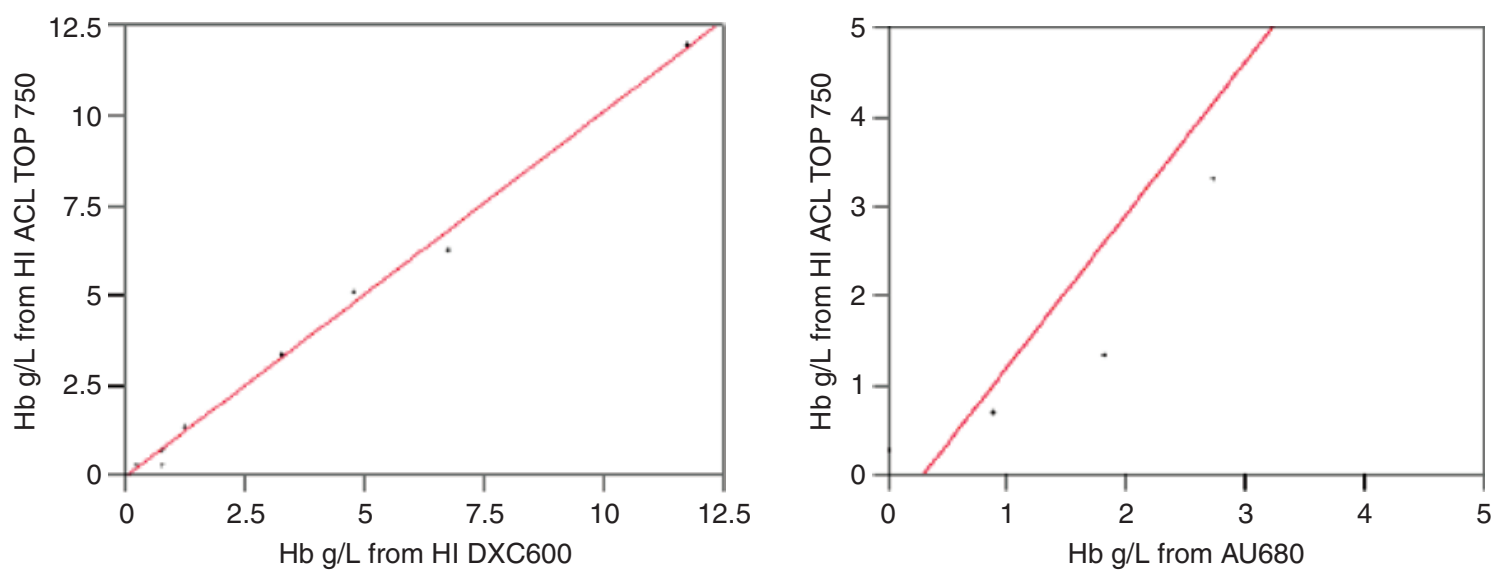

Figure 5: Correspondence between free- $\mathrm{Hb}_{(\mathrm{g} / \mathrm{L})}$ measured on the ACL TOP 750 and DXC600 systems $\left(\mathrm{Hb}_{(\mathrm{g} / \mathrm{L})} \mathrm{ACL}\right.$ TOP $750=-0.04+1.01 \mathrm{Hb}(\mathrm{g} / \mathrm{L})$ DXC600, $r^{2}=0.999$ ).

An unsatisfactory correspondence was found between $\mathrm{Hb}_{(\mathrm{g} / \mathrm{L})}$ measured on the ACL TOP 750 system and $\mathrm{HI}$ measured on the AU680 system $\left(\mathrm{Hb}_{(\mathrm{g} / \mathrm{L})}\right.$ ACL TOP $750=-0.49+1.70 \mathrm{Hb}_{(\mathrm{g} / \mathrm{L})}$ AU680, $\left.\mathrm{r}^{2}=0.866\right)$. 


\section{Correlation between free- $\mathrm{Hb}$ results measured by different methods}

The free-Hb SMM, performed according to the protocol of Fairbanks et al. [11], was chosen as the reference method. Linear regressions were performed between SMM and each other system (Figure 6).

On comparing DXC600 and SMM, an $\mathrm{r}^{2}=0.893$ was obtained (HI DXC600 $=-0.10+1.27$ free- $\mathrm{Hb}$, where -0.10 was the intercept and 1.27 was the slope of the regression line). Bland-Altman analysis defined a bias of $0.13 \mathrm{~g} / \mathrm{L}$ (confidence interval [CI] 95\%: 0.09-0.16), with an agreement ranging from -0.46 to 0.71 . The plot showed a slight overestimation of DXC600 values with respect to SMM and a moderate positive trend of the differences; the bias seemed to change with $\mathrm{Hb}$ concentration (Figure 6A).

Linear regression between AU680 and SMM gave an $\mathrm{r}^{2}=0.853$ (HI AU680 $=0.06+1.06$ free-Hb); Bland-Altman analysis quantified a bias of $0.11 \mathrm{~g} / \mathrm{L}$ (CI 95\%: 0.08-0.14) with limits of agreement ranging from -0.40 to 0.62 (Figure 6B).

Results from ACL TOP 750 and SMM were related in a linear regression with an $\mathrm{r}^{2}=0.917$ (HI TOP $750=0.02+1.26$
A

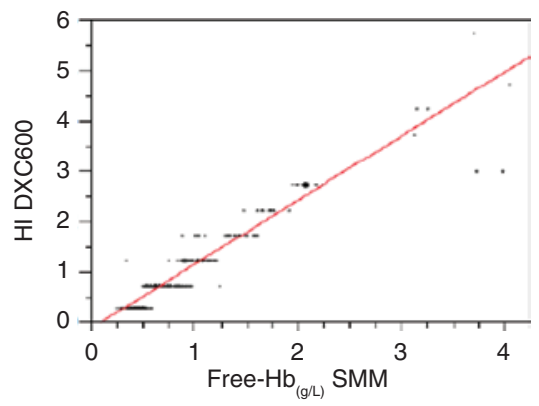

B

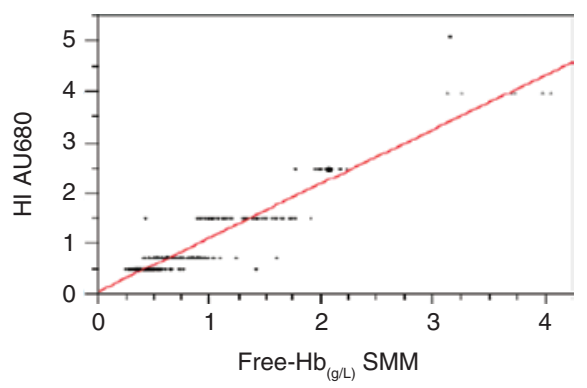

C

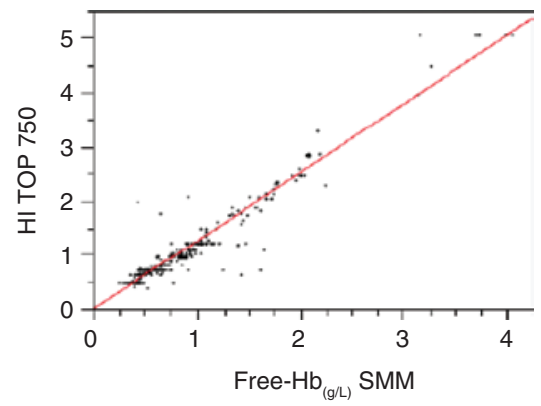

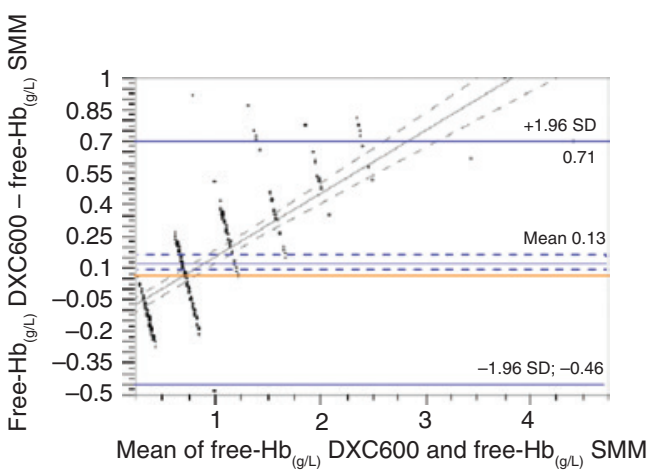
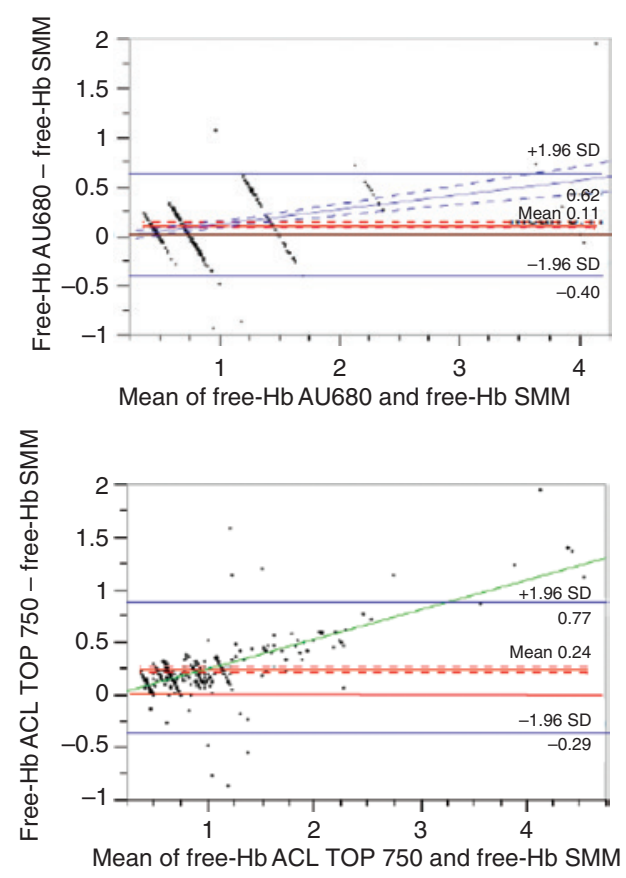

Figure 6: Linear regressions and Bland-Altman plots between hemolysis indexes (HIs) obtained by the spectrophotometric method (SMM) and other measuring systems.

(A) For DXC600, $\mathrm{r}^{2}=0.893(\mathrm{HI} \mathrm{DXC} 600=-0.10+1.27$ free- $\mathrm{Hb})$. Bland-Altman analysis defined a mean bias of $0.13 \mathrm{~g} / \mathrm{L}(\mathrm{Cl} 95 \%$ : 0.09-0.16), with an agreement ranging from -0.46 to 0.71. (B) For AU680, $\mathrm{r}^{2}=0.853$ (HI AU680=0.06 +1.06 free-Hb). Bland-Altman analysis showed a bias of $0.11 \mathrm{~g} / \mathrm{L}(\mathrm{Cl} 95 \%$ : 0.08-0.14) with limits of agreement ranging from -0.40 to 0.62 . (C) For $\mathrm{ACL}$ TOP 750 , $\mathrm{r}^{2}=0.917(\mathrm{HI}$ TOP $750=0.02+1.26$ free-Hb). Bland-Altman analysis defined a bias of $0.24 \mathrm{~g} / \mathrm{L}(\mathrm{Cl} 95 \%$ : $0.21-0.27)$, with an agreement ranging from -0.29 to 0.77 . 

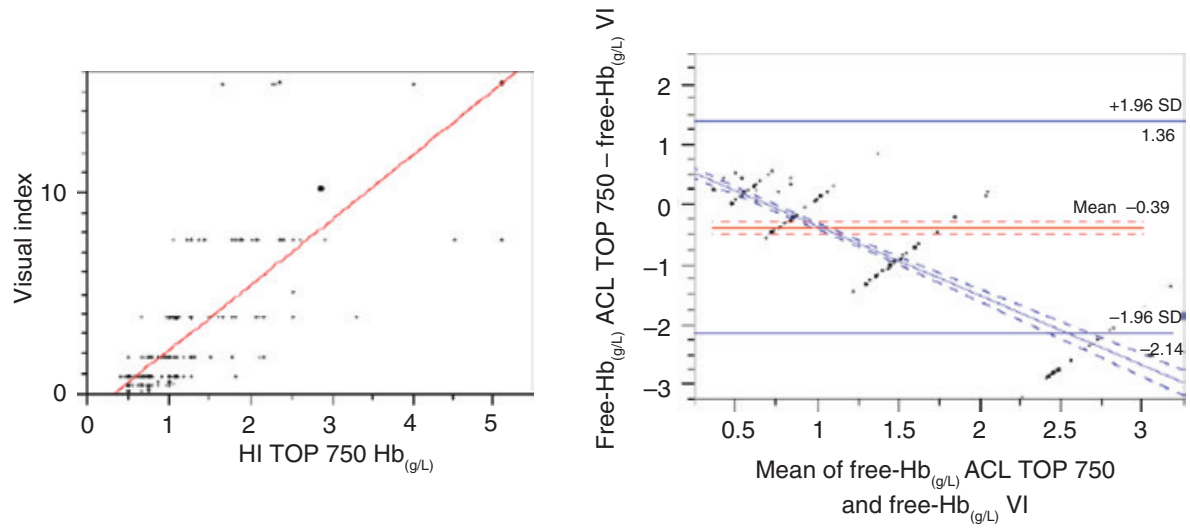

Figure 7: Linear regression analysis between visual index $(\mathrm{VI})$ and $\mathrm{HI}$ on the $\mathrm{ACL}$ TOP 750 system resulted in an $\mathrm{r}^{2}=0.648, \mathrm{VI}=-1.02+3.22$ HI TOP 750.

Bland-Altman analysis showed a bias of -0.39 (CI 95\%: -0.49 to -0.30$)$, with limits of agreement from -2.14 to 1.36 .

free-Hb). Bland-Altman analysis defined a bias of $0.24 \mathrm{~g} / \mathrm{L}$ (CI 95\%: 0.21-0.27), with an agreement ranging from -0.29 to 0.77 ; the bias seemed to increase with $\mathrm{Hb}$ concentration for ACL TOP 750 as well, being lower when concentrations were lower. For the ACL TOP 750 and SMM comparison, results from 12 of 306 samples fell outside the limits of agreement (4\%; Figure 6C).

Linear regression analysis was performed between results from visual index (VI) and the ACL TOP 750 system $\left(\mathrm{r}^{2}=0.648, \mathrm{VI}=-1.02+3.22 \mathrm{HI}\right.$ ACL TOP 750; Figure 7). Bland-Altman analysis defined a bias of $-1.41 \mathrm{~g} / \mathrm{L}$ (CI 95\%: -1.13 to -1.70 ) and the limits of agreement ranged from -6.32 to 3.48. Limiting the Bland-Altman analysis to a free- $\mathrm{Hb}$ level $<3 \mathrm{~g} / \mathrm{L}$, in the range of critical values to verify sample conformity in the hemostasis laboratory, the mean bias changed to -0.39 (CI 95\%: -0.49 to -0.30 ), with an agreement ranging from -2.14 to 1.36 . The plot showed a negative trend according to $\mathrm{Hb}$ level; at low $\mathrm{Hb}$ concentration, VI overestimated free-Hb with respect to the automated ACL TOP 750 system, and the overestimation increased with $\mathrm{Hb}$ levels (Figure 7).

Multivariate analysis showed the best correlation with the reference method for the ACL TOP 750 system $\left(r^{2}=0.958\right)$, followed by DXC600 $\left(r^{2}=0.945\right)$ and AU680 $\left(r^{2}=0.923\right)$; the VI gave an unsatisfactory correlation grade $\left(r^{2}=0.796\right)$. Sensitivity, specificity and diagnostic accuracy were calculated for each method. For the HIL system on ACL TOP 750, we obtained a sensitivity and a specificity of 0.939 and 0.934 , respectively, with a diagnostic accuracy of $94 \%$. For HI scores on the DXC600 system, the sensitivity was 0.939 , the specificity was 0.927 and the diagnostic accuracy was $93 \%$. HI scores on the AU680 system showed a sensitivity of 0.970 , a specificity of 0.828 and a diagnostic accuracy of $84 \%$.

\section{Discussion}

The analytical performance of automated methods for measuring hemolysis in plasma sodium citrate samples in hemostasis laboratories was assessed. The ability of the ACL TOP 750 HIL system in discriminating and classifying different degrees of hemolysis was compared with a manual spectrophotometric method [11] and a visual method [14].

Unlike other studies, individual specimens were used as they were normally received in the laboratory for coagulation analysis, rather than as pooled samples.

The visual hemolysis assessment was confirmed to be too subjective; a poor correlation between VI and ACL TOP $750 \mathrm{HI}$ was found, possibly due to the difficulty in discriminating mild hemolysis only by visual impression. Bland-Altman analysis showed an overestimation of VI with respect to the automated system, ACL TOP 750, indicating poor discrimination of hemolysis by human eyes.

We were interested in evaluating the difference in the number of hemolyzed samples rejected on the basis of visual inspection compared to an automated method. We classified samples as "to be processed" or "to be rejected" based on an arbitrary free- $\mathrm{Hb}$ threshold of $0.15 \mathrm{~g} / \mathrm{L}$. According to our laboratory experience with coagulation tests, this was a level above which a sample could not be reliably assayed, and represented the mean value of the slightly hemolyzed sample category in the classification of hemolysis [3, 13]. It was also very close to the threshold of $\sim 0.20 \mathrm{~g} / \mathrm{L}$ at which hemolysis was visible to the naked eye in both plasma and serum [12]. In addition, at a level slightly higher than the normal plasma value of $0.13 \mathrm{~g} / \mathrm{L}$ [9], it represented a useful cut-off for evaluating systems that must distinguish between normal and hemolyzed samples. 
Based on visual classification, 150 of 306 (49\%) samples would be discarded; however, based on the HIL check on the ACL TOP 750 system, only 49 of 306 (16\%) samples were not suitable for analysis, of which 48 were present in the group of 150 that were discarded based on visual examination (98\%). For the visual method, the $0.15 \mathrm{~g} / \mathrm{L}$ cut-off was not applicable, being too narrow and low. Sample rejection occurred when the degree of hemolysis on the Luksic visual scale was $\geq 2$, i.e. when free-Hb was $>0.5 \mathrm{~g} / \mathrm{L}$ [14]. The sensitivity for VI was 0.970 , but the specificity was 0.568 and we found a poor diagnostic accuracy (61\%). If the evaluation of sample conformity in a hemostasis laboratory was based only on visual inspection, a large number of samples would be discarded, most with hemolysis at acceptable levels for blood assays. Thus, we confirmed that visual assessment of hemolysis degree should be replaced with automatic detection $[2,10]$.

Analysis of variance (ANOVA) for paired data showed no correlation between $\mathrm{HI} \geq 0.15 \mathrm{~g} / \mathrm{L}$ and the sex and age of patients; however, there was a slight association with the departments from which the samples were collected $(p<0.05)$. The higher prevalence of hemolyzed samples from emergency departments is in agreement with previous reports [3]. Among all hemolyzed samples, 56\% were recorded from non-hospitalized patients. This finding could be explained by the significant number (89\%) of non-hospitalized patients undergoing coagulation tests being on oral anticoagulant therapy, and in this context venous access is very difficult. In addition, samples must be delivered to the laboratory from patients' homes and transport conditions may vary and affect their suitability for testing.

\section{Conclusions}

All the methods for measuring the degree of hemolysis in a sample, both automated and manual spectrophotometric approaches, demonstrated satisfactory analytical performances. In particular, the ACL TOP 750 system showed an excellent correspondence with the spectrophotometric reference method, and good sensitivity, specificity and accuracy. The study confirmed that coagulation and hemostasis laboratories need to use objective and standardized systems to quantify sample hemolysis. Visual inspection alone may cause the unnecessary rejection of about $50 \%$ of samples, $35 \%$ of which could be processed quite adequately if an automated and standardized method was used.
Acknowledgments: We sincerely thank Dr. Susan Elizabeth Gwynne and Dr. Karin Tyack for their revision of the text.

Author contributions: All the authors have accepted responsibility for the entire content of this submitted manuscript and approved submission.

Research funding: None declared.

Employment or leadership: None declared.

Honorarium: None declared.

Competing interests: The funding organization(s) played no role in the study design; in the collection, analysis, and interpretation of data; in the writing of the report; or in the decision to submit the report for publication.

\section{References}

1. Lippi G, Baird GS, Banfi G, lenius KB, Cadamuro J, Church S, et al. Improving quality in the preanalytical phase through innovation, on behalf of the European Federation for Clinical Chemistry and Laboratory Medicine (EFLM) Working Group for Preanalytical Phase (WG-PRE) Medicine (EFLM) Working Group for Preanalytical Phase (WG-PRE). Clin Chem Lab Med 2017;55:489-500.

2. Lippi G, Cadamuro J, von Meyer A, Simundic A. Pratical recommendations for managing hemolyzed samples in clinical chemistry testing. Clin Chem Lab Med 2018;56:718-27.

3. Lippi G, Cervellin G, Favaloro EJ, Plebani M. In vitro and in vivo hemolysis. An unresolved dispute in laboratory medicine. Berlin, Germany: Walter de Gruyter GmbH; 2012.

4. Simundic AM, Bölenius K, Cadamuro J, Church S, Cornes MP, van Dongen-Lases EC, et al. Joint EFLM-COLABIOCLI. Recommendation for venous blood sampling. Clin Chem Lab Med 2018;56:2015-38

5. CLSI H21-A5 Clinical and Laboratory Standards Institute. Collec tion, transport, and processing of blood specimens for testing plasma-based coagulation assays; approved guidelines $5^{\text {th }}$ ed. 2008.

6. Woolley A, Golmard JL, Kitchen S. Effects of haemolysis, icterus and lipaemia on coagulation tests as performed on Stago STA-Compact-Max analyser. Int J Lab Hematol 2016;38:375-88.

7. Hernaningsih Y, Akualing JS. The effects of hemolysis on plasma prothrombin time and activated partial thromboplastin time tests using photo-optical method. Medicine (Baltimore) 2017;96:e7976.

8. Lippi G, Ippolito L. Interference of spurious hemolysis on prothrombin time, activated partial thromboplastin time and fibrinogen. NZ J Med Lab Science 2014;68:52-4.

9. Lippi G, Cadamuro J. Visual assessment of sample quality: quo usque tandem? Clin Chem Lab Med 2018;56:513-5.

10. CLSI document C56-A Clinical and Laboratory Standards Institute. Hemolysis, icterus, and lipemia/turbidity indices as indicators of interference in clinical laboratory analysis; approved guideline. Wayne, PA, USA: Clinical and Laboratory Standards Institute; 2012. 
11. Fairbanks VF, Ziesmer SC, O'Brien PC. Methods for measuring plasma hemoglobin in micromolar concentration compared. Clin Chem 1992;38:132-40.

12. Dolci A, Panteghini M. Harmonization of automated hemolysis index assessment and use: is it possible? Clinica Chimica Acta 2014;432:38-43.

13. Nagant C, Rozen L, Demulder A. HIL interferences on three hemostasis analyzers and contribution of a preanalytical module for routine coagulation assays. Clin Lab 2016;62:1979-87.

14. Luksic AH, Gabaj NN, Miler M, Dukic L, Bakliza A, Simundic AM. Visual assessment of hemolysis affects patient safety. Clin Chem Lab Med 2018;56:574-81.

15. Lippi G, Ippolito L, Favaloro EJ. Technical evaluation of the novel preanalytical module on instrumentation laboratory ACL
TOP: advancing automation in hemostasis testing. J Lab Autom 2016;18:382-90.

16. Kwoun WJ, Ahn JY, Park PW, Seo YH, Kim KH, Seo JY, et al. Performance evaluation of the preanalytic module of the ACL TOP 750 hemostasis lab system. Ann Lab Med 2018;38:484-6.

17. CLSI H21-A5: Collection. Transport, and processing of blood specimens for testing plasma-based coagulation assays and molecular hemostasis assays, $5^{\text {th }}$ ed. 2008.

18. CLSI EP5-A2 protocol. Evaluation of precision performance of quantitative measurement methods; approved guideline second edition, vol. 24, 2004 (Wayne, PA).

19. CLSI EP17-A protocol. Protocols for determination of limits of detection and limits of quantification; approved guidelines, vol. 24, 2004 (Wayne, PA). 